\title{
Buccal Mucosa Verrucous Carcinoma
}

National Cancer Institute

\section{Source}

National Cancer Institute. Buccal Mucosa Verrucous Carcinoma. NCI Thesaurus. Code C8175.

A verrucous carcinoma of the oral cavity that arises from the buccal mucosa. 